\title{
Dedicated cardiac cameras: a new option for nuclear myocardial perfusion imaging
}

\author{
Orazio Schillaci • Roberta Danieli
}

Published online: 1 July 2010

(C) Springer-Verlag 2010

\begin{abstract}
Among non-invasive methods for the assessment of ischaemic heart disease, myocardial perfusion imaging (MPI) using SPECT tracers is firmly established; it allows one to obtain quantifiable and cost-effective information for the management of patients with suspected or proven coronary artery disease (CAD). In clinical practice, MPI for evaluating regional myocardial blood flow and viability under rest or stress conditions has emerged with two clear roles: diagnosis of CAD and assessment of prognosis in patients with known CAD [1]. In fact, MPI provides clinical benefit in the initial evaluation of patients with suspected, but unproven $\mathrm{CAD}$, and in those patients where a diagnosis of CAD has been established and prognostic information on long-term outcomes is required, also for making the most appropriate treatment decisions.

The evolution of MPI over the years has occurred in parallel with advances in instrumentation, particularly with the transformation from planar gamma camera imaging to tomographic images with SPECT technology, which now includes gated acquisition for combined assessment of myocardial perfusion and left ventricular function. Never-
\end{abstract}

This Editorial Commentary refers to the article http://dx.doi.org/ 10.1007/s00259-010-1441-1

O. Schillaci $\cdot$ R. Danieli

Department of Biopathology and Diagnostic Imaging,

University "Tor Vergata",

Rome, Italy

O. Schillaci

IRCCS Neuromed,

Pozzilli, Italy

O. Schillaci $(\bowtie)$

Viale G. Mazzini 121,

00195 Rome, Italy

e-mail: orazio.schillaci@uniroma2.it theless, MPI is still affected by several drawbacks that are inherent to any nuclear technique with low photon flux, like attenuation due to non-uniform tissue, motion artefacts due to time-consuming scan and radiation exposure [2]. Therefore, shortening scan acquisition and reducing radiation dose without decreasing image quality are of the utmost importance for nuclear MPI in maintaining its central role in the management of patients with suspected or known CAD, especially in an era of impressive advances in coronary computed tomography (CT), cardiac magnetic resonance and ultrasound imaging.

The dedicated solid-state cardiac camera used by Ben-Haim et al. [3] in their study published in this issue of the European Journal of Nuclear Medicine and Molecular Imaging represents an important answer to the above-mentioned clinical needs for nuclear MPI.

Moreover, various new dedicated hardware camera systems with optimized acquisition geometry, collimator design and associated reconstruction software have been recently introduced by several manufacturers [2, 4-7]. These cameras vary in the number and type of scanning or stationary detectors and in whether $\mathrm{NaI}$ or cadmium zinc telluride (CZT) solid-state detectors are employed. Nevertheless, they all have in common the ability to image just the cardiac field of view and to increase sampling of the myocardial region, with the possibility of enhancing count sensitivity from fivefold to tenfold, combined with an improvement also in spatial resolution. The increased sensitivity of these systems allows faster imaging time that significantly reduces patient motion during acquisition, which is favourably affected also by a more comfortable upright or reclining positioning, without the need of putting the arms above the head.

The diagnostic performance for nuclear MPI of one of these ultrafast cardiac gamma cameras with CZT solid-state 
semiconductor detectors (Discovery NM 530c, GE Healthcare) has been recently evaluated in a group of 75 patients and compared with the outcomes of a conventional dualdetector SPECT camera [8]. All patients-referred to MPI for suspected CAD, follow-up of a known CAD or preoperative risk assessment before major non-cardiac surgery-underwent a 1 -day ${ }^{99 \mathrm{~m}} \mathrm{Tc}$-tetrofosmin adenosine or dobutamine stress/rest imaging protocol, according to the European Association of Nuclear Medicine (EANM) guidelines [9]. Electrocardiogram-gated images were first acquired on a conventional gamma camera with a $15-\mathrm{min}$ scan time each for stress and rest, and then they were immediately repeated on an ultrafast CZT camera with a 3-min acquisition time for stress and a 2-min time for rest. MPI reconstruction for both standard SPECT and CZT images were made on a dedicated workstation, using a standard iterative reconstruction algorithm for the first ones and a new dedicated iterative algorithm for the ultrafast acquisitions. Quantitative analysis of segmental myocardial uptake values (per cent of maximum) was performed on MPI polar maps with a 20-segment model for the left ventricle.

Image quality was assessed by visual analysis with good results in $16 \%$ and excellent in $84 \%$ of patients for SPECT compared to good in $15 \%$ and excellent in $85 \%$ of cases for the CZT scans. There was excellent clinical agreement (normal, ischaemia, scar) between CZT and conventional SPECT, both on a per-patient basis $(96 \%)$ and on a pervessel territory basis $(96.4 \%)$, as indicated by a highly significant correlation between segmental tracer uptake values $(p<0.001)$ using SPECT MPI as the reference standard. Moreover, also left ventricular ejection fraction values calculated with the two imaging systems demonstrated high correlation $(p<0.001)$. The findings of this preliminary study clearly suggest that the new dedicated CZT camera is able to obtain an image quality comparable to that of state-of-the-art SPECT cameras, providing equivalent clinical information, with an important more than fivefold decrease of acquisition time.

Similar findings, indicating a diagnostic performance of the same dedicated cardiac camera comparable to that of a standard dual-detector SPECT on a per-patient basis with significantly shorter acquisition times, have been obtained by Esteves et al. [10] in a multicentre trial designed to compare the two imaging systems in a group of 168 patients undergoing 1-day ${ }^{99 \mathrm{~m}}$ Tc-tetrofosmin ECG-gated rest/stress (exercise, adenosine or dipyridamole) MPI. The same injected doses were used for performing all the images, with CZT acquisitions obtained immediately prior to conventional SPECT ones. Rest and stress acquisition times were 4 and 2 min for the cardiac camera and 14 and $12 \mathrm{~min}$ for standard SPECT. All scans were visually evaluated on a patient level and on a vascular territory level using a standard 5-point scale. Agreement between CZT camera and SPECT for presence or absence of myocardial perfusion defects on a per-patient analysis was 91.9 and $92.5 \%$, respectively. The overall agreement rate (defect present or absent) was $96 \%$ both in the left anterior descending artery and in left circumflex artery territories, and $93 \%$ in the distribution of the right coronary artery. Rest and stress left ventricular ejection fractions and volumes between the two imaging systems were significantly correlated. It is worth noting that superior image quality was observed in this series with the dedicated camera: in fact, rest images were considered excellent in $83 \%$ of cases compared to $70 \%$ of SPECT rest images and stress images were considered excellent in $86 \%$ of cases compared to $68 \%$ of SPECT stress images.

Herzog et al. [11] in a recent study evaluated the optimal scan time for MPI of this ultrafast camera in a group of 20 patients undergoing a 1-day ${ }^{99 \mathrm{~m}} \mathrm{Tc}$-tetrofosmin adenosine stress/rest protocol, using standard doses. Just after the acquisition of images by means of a conventional dual-head SPECT camera, all scans were immediately repeated on the CZT camera over a 6-min acquisition time and reconstructed from list-mode raw data to obtain scan durations from $1 \mathrm{~min}$ up to a maximum of $6 \mathrm{~min}$. The minimal required scan times for the ultrafast system, which allows excellent agreement with a standard dual-detector SPECT camera, with regard both to uptake and clinical findings, were $3 \mathrm{~min}$ for a low dose (i.e. $300-350 \mathrm{MBq}$ at stress) and 2 min for a high one (two to three times the stress dose injected at rest), respectively.

The system used in the above-mentioned three studies $[8,10,11]$ is based on Alcyone technology (GE Healthcare), which combines focused pinhole collimation, an array of CZT pixilated detectors, 3-D reconstruction and stationary data acquisition. In this cardiac camera, the detectors and collimators do not move during image acquisition and all lines of response are acquired simultaneously through a proprietary multi-pinhole collimator. Also the D-SPECT camera (Spectrum Dynamics) employed by Ben-Haim et al. [3] uses solid-state detectors in the form of CZT, which improves the energy and spatial resolutions, thus facilitating the development of simultaneous dualisotope imaging protocols. The pixilated CZT detector arrays are mounted in nine vertical columns and placed in a $90^{\circ}$ gantry geometry. Each of the nine detector columns is fitted with a tungsten, parallel-hole square collimator, and it is fixed in a mechanical mounting, with the data acquisition performed by rotating these multiple columns in synchrony while all nine are simultaneously imaging the heart. Images are performed after obtaining a 1-min scout scan for the nine detectors to identify the location of the heart to set the limits of each detector's fanning motion, and then they are reconstructed by a modified iterative algorithm which 
mathematically models the acquisition and collimator geometry [12].

In a previous pilot clinical study, the performance of $\mathrm{D}$ SPECT had been evaluated in 44 patients and compared with the results of conventional SPECT imaging [13]. This group of patients was referred for 1-day stress (exercise or dipyridamole)/rest ${ }^{99 \mathrm{~m}} \mathrm{Tc}$-sestamibi gated MPI; they had low pretest likelihood of $\mathrm{CAD}$, moderate to high pretest likelihood of $\mathrm{CAD}$ in the absence of documented $\mathrm{CAD}$ or history of myocardial infarction. D-SPECT images were performed within 30 min after standard SPECT; stress and rest acquisition times were 16 and 12 min for conventional imaging and 4 and 2 min for D-SPECT, respectively. All MPIs were visually analysed and semi-quantitatively scored with a 20 -segment model of the left ventricle and a 5-point scale; the summed stress score (SSS) and summed rest score (SRS) were calculated; also image quality and diagnostic confidence were assessed. Moreover, myocardial counts/min were calculated for both conventional SPECT and D-SPECT.

The findings of this study indicated excellent linear correlation between both SSS and SRS by conventional SPECT versus SSS and SRS by dedicated cardiac camera $(p<0.00001)$. All of the patients with low prescan likelihood of CAD had normal stress perfusion by both cameras; moreover, there was a good correlation between MPI findings by both cameras compared with coronary angiography in the seven patients who underwent this procedure within 60 days after the nuclear imaging. Image quality was graded as good or higher in $96.3 \%$ of cases for D-SPECT and standard camera. Diagnostic confidence in image interpretation was also similar for the two systems. Finally, myocardial count rate was significantly higher (seven- to eightfold) in D-SPECT compared with conventional SPECT both at stress and rest.

The D-SPECT camera system had been previously described and validated in a paper using simulation, various physical phantoms and pilot patient data [14]. In fact, various phantom studies were performed comparing the new technology with conventional SPECT camera, including measurements of line sources and single- and dualradionuclide studies of a torso phantom; moreover, simulations were also performed using a cardiothoracic phantom. Lastly, 18 patients were scanned with both cameras. The findings of this study indicated that the D-SPECT had a count sensitivity ten times higher than that of the conventional camera and a moderately compensated spatial resolution. These capabilities allowed the new imaging system to obtain 2-min clinical images with good to excellent quality and improved myocardial edge definition. Furthermore, dualradionuclide studies with a phantom showed the new potential of the dedicated cardiac camera for a two-tracer simultaneous acquisition.
The characteristics of these gamma cameras with semiconductor detectors can allow one to evaluate new MPI protocols which may offer advantages over the standard protocols used till now. With this aim, Berman et al. [15] have evaluated 374 consecutive patients using a rapid stress ${ }^{201} \mathrm{Tl} /$ rest ${ }^{99 \mathrm{~m}} \mathrm{Tc}$-sestamibi or -tetrofosmin protocol, which was finished in about $20 \mathrm{~min}$. At peak stress, patients were injected with ${ }^{201} \mathrm{Tl}$ and after a 5-min post-stress monitoring period, supine and sitting acquisitions were performed sequentially; just after, ${ }^{99 \mathrm{~m}} \mathrm{Tc}$-sestamibi or tetrofosmin was injected and a single 4-min sitting rest scan was acquired. The injected activities were $2.2 \pm 0.3 \mathrm{mCi}$ for ${ }^{201} \mathrm{Tl}, 8.9 \pm 1.7 \mathrm{mCi}$ for sestamibi and $8.8 \pm 2.6 \mathrm{mCi}$ for tetrofosmin, respectively, with an effective dose of $11.9 \mathrm{mSv}$. Image quality was good or excellent in $96 \%$ of patients, with rest scans assessed in the same way in $99 \%$ of cases, despite beginning only $2 \mathrm{~min}$ after injection. Therefore, the rapid stress ${ }^{201} \mathrm{Tl} /$ rest ${ }^{99 \mathrm{~m}} \mathrm{Tc}$ protocol for use with high-speed MPI demonstrated image quality and radiation dosimetry similar to those observed with a conventional rest/stress ${ }^{99 \mathrm{~m}} \mathrm{Tc}$ protocol. Furthermore, thanks to its spectral resolution, the CZT camera has the potential to simultaneously record dual tracers.

This opportunity has been then specifically evaluated by Ben-Haim et al. in their paper published in this issue of the European Journal of Nuclear Medicine and Molecular Imaging. The study included 24 patients with ischaemic heart disease who were injected at rest with ${ }^{201} \mathrm{Tl}(80 \mathrm{MBq})$ and with ${ }^{99 \mathrm{~m}} \mathrm{Tc}-$ sestamibi $(250 \mathrm{MBq})$ after adenosine pharmacologic stress. Both image quality and accuracy of simultaneous dual-radionuclide MPI acquired with the D-SPECT were compared with sequential imaging performed with a standard dual-head gamma camera. The protocol for the CZT camera included a 6-min list-mode gated rest ${ }^{201} \mathrm{Tl}$ acquisition just after conventional rest scan and a 15-min list-mode simultaneous dual-radionuclide (rest and stress) gated images. The authors used a specifically designed spillover and scatter correction method for processing the data obtained with the dedicated camera.

It is worth noting that simultaneous D-SPECT images were scored as good or very good in all of the 24 rest components and in 21 of the 24 stress components of the study, whereas rest ${ }^{201} \mathrm{Tl}$ images were evaluated as good/ very good in all of the 22 cases considered. The SSS and SRS of standard SPECT and dual-radionuclide D-SPECT were highly correlated, as well as the SRS of dualradionuclide D-SPECT and ${ }^{201} \mathrm{Tl} \mathrm{D}$-SPECT. In particular, 9 rest and 17 stress studies were abnormal on both conventional and CZT MPI; however, in 6 patients, the stress perfusion defects were significantly larger on dualradionuclide D-SPECT images than on standard MPI. These outcomes clearly indicate that D-SPECT allows a 
single imaging session including fast and high-quality simultaneous ${ }^{201} \mathrm{Tl} /{ }^{99 \mathrm{~m}} \mathrm{Tc}$-sestamibi stress/rest MPI, with similar quality of images and diagnostic accuracy with respect to standard SPECT MPI.

The very fast acquisition capability of D-SPECT was employed by Sheikine et al. [16] to study rapid changes in radiotracer distribution. They have recently described two cases of patients with angiographically proved CAD, in whom ${ }^{99 \mathrm{~m}} \mathrm{Tc}$-sestamibi showed a notable redistribution between early $(6-8 \mathrm{~min})$ and late $(60-70 \mathrm{~min})$ post-stress imaging. Early post-stress D-SPECT images were consistent with the findings on coronary angiography. These results suggest that early imaging may be more sensitive for CAD detection, with a significant underestimation of the extent and severity of ischaemia on late images. However, it is worth noting that high-quality "early" imaging in these patients was allowed by the increased sensitivity of D-SPECT, which is able to perform stress images in only 2 min and, so, to permit serial acquisitions at several time points after the stress test.

The results obtained in these first studies using the dedicated cardiac cameras clearly offer great promise for their clinical applications in nuclear cardiology. Compared with conventional SPECT imaging, these new systems are able to significantly reduce MPI times, which will result in improving patient comfort and thus in causing fewer motion artefacts. Moreover, these highspeed cameras are able to provide images comparable to those obtained with standard SPECT, with the same diagnostic outcomes. It is of the utmost importance to highlight that these new devices can maintain MPI quality while reducing administered activity and radiation dose, which are two fundamental aspects especially in these times characterized by ${ }^{99 \mathrm{~m}} \mathrm{Tc}$ shortage and concerns about the increasing number of patients being exposed for diagnostic purposes to radiation.

In conclusion, the advent of these ultrafast cameras can really improve the potential of MPI, also introducing new imaging protocols with simultaneous acquisition of dual tracers, but larger studies comparing their results with coronary angiography and/or coronary $\mathrm{CT}$ are needed to finally establish their true diagnostic accuracy and clinical usefulness.

\section{References}

1. Russel III RR, Zaret BL. Nuclear cardiology: present and future. Curr Probl Cardiol 2006;31:557-629.

2. Slomka PJ, Patton JA, Berman DS, Germano G. Advances in technical aspects of myocardial perfusion SPECT imaging. J Nucl Cardiol 2009;16:255-76.

3. Ben-Haim S, Kacperski K, Hain S, Van Gramberg D, Hutton BF, Erlandsson K, et al. Simultaneous dual-radionuclide myocardial perfusion imaging with a solid-state dedicated cardiac camera. Eur J Nucl Med Mol Imaging 2010. doi: 10.1007/S00259-010-1441-1

4. Madsen MT. Recent advances in SPECT imaging. J Nucl Med 2007;48:661-73.

5. Patton JA, Slomka PJ, Germano G, Berman DS. Recent technologic advances in nuclear cardiology. J Nucl Cardiol 2007;14:501-13.

6. Garcia EV, Faber TL. New trends in camera and software technology in nuclear cardiology. Cardiol Clin 2009;27:227-36.

7. Nichols KJ, Van Tosh A, Palestro CJ. Prospects for advancing nuclear cardiology by means of new detector designs. J Nucl Cardiol 2009;16:691-6.

8. Buechel RR, Herzog BA, Husmann L, Burger IA, Pazhenkottil AP, Treyer V, et al. Ultrafast nuclear myocardial perfusion imaging on a new gamma camera with semiconductor detector technique: first clinical validation. Eur J Nucl Med Mol Imaging 2010;37:773-8.

9. Hesse B, Tägil K, Cuocolo A, Anagnostopoulos C, Bardiés M, Bax J, et al. EANM/ESC procedural guidelines for myocardial perfusion imaging in nuclear cardiology. Eur J Nucl Med Mol Imaging 2005;32:855-97.

10. Esteves FP, Raggi P, Folks RD, Keidar Z, Askew JW, Rispler S, et al. Novel solid-state-detector dedicated cardiac camera for fast myocardial perfusion imaging: multicenter comparison with standard dual detector cameras. J Nucl Cardiol 2009;16:927-34.

11. Herzog BA, Buechel RR, Katz R, Brueckner M, Husmann L, Burger IA, et al. Nuclear myocardial perfusion imaging with a cadmium-zinc-telluride detector technique: optimized protocol for scan time reduction. J Nucl Med 2010;51:46-51.

12. Erlandsson K, Kacperski K, van Gramberg D, Hutton BF. Performance evaluation of D-SPECT: a novel SPECT system for nuclear cardiology. Phys Med Biol 2009;54:2635-49.

13. Sharir T, Ben-Haim S, Merzon K, Prochorov V, Dickman D, BenHaim S, et al. High-speed myocardial perfusion imaging initial clinical comparison with conventional dual detector anger camera imaging. JACC Cardiovasc Imaging 2008;1:156-63.

14. Gambhir SS, Berman DS, Ziffer J, Nagler M, Sandler M, Patton J, et al. A novel high-sensitivity rapid-acquisition single-photon cardiac imaging camera. J Nucl Med 2009;50:635-43.

15. Berman DS, Kang X, Tamarappoo B, Wolak A, Hayes SW, Nakazato R, et al. Stress thallium-201/rest technetium-99m sequential dual isotope high-speed myocardial perfusion imaging. JACC Cardiovasc Imaging 2009;2:273-82.

16. Sheikine Y, Berman DS, Di Carli MF. Technetium-99m-sestamibi redistribution after exercise stress test identified by a novel cardiac gamma camera: two case reports. Clin Cardiol 2010;33:E39-45. 